\title{
Momentum paradox in a vortex core
}

\author{
Roberta Zambrini ${ }^{1 *}$, Laura C. Thomson ${ }^{1}$, Stephen M. Barnett ${ }^{1}$, \\ Miles Padgett ${ }^{2}$ \\ 1 Department of Physics, University of Strathclyde, \\ Glasgow G4 0NG, UK. \\ 2 Department of Physics and Astronomy University of Glasgow, \\ Glasgow G12 8QQ, UK.
}

september 14, 2004

\begin{abstract}
The identification of angular momentum $l \hbar$ per photon with optical vortices of charge $l$ appears to require that the field amplitude be zero within a finite distance of the vortex. This, however, is not compatible with the known form of beams such as the Laguerre-Gaussian and Bessel beams. We resolve this paradox by analysing the propagation of a Bessel beam through a small circular aperture, showing that the resulting field has evanescent components.
\end{abstract}

*roberta@phys.strath.ac.uk, tel: 0141548 3376, fax: 01415522891 


\section{Introduction and paradox}

It is now well established that optical beams with helical wavefronts carry orbital angular momentum. In particular, a beam with azimuthal phase dependence $e^{i l \phi}$ has an associated orbital angular momentum of $l \hbar$ per photon $[1,2]$. This azimuthal phase dependence is undefined on the beam axis and hence the associated amplitude of the field will be zero. The field-zero is a beam dislocation or optical vortex and such features have played an important role in the recent development of optics [3]. Although the field is zero at the centre of the vortex, it will have a non-zero value around it, typically growing in amplitude as the

$|l|^{t h}$ power of the distance from the vortex core for small distances. We present an argument, based on the orbital angular momentum of light, that questions the existence of this non-zero amplitude in the immediate vicinity of the vortex core. Our resolution of this paradox requires us to examine the propagation of the light near to the core.

Our paradox may be described either within classical or quantum optics, but it is most simply stated in terms of photons. Consider a monochromatic beam of angular frequency $\omega=c k_{0}$. Each photon comprising the beam will have energy $\hbar \omega$. If we consider a plane wave then it will also carry linear momentum

$$
\vec{p}=\hbar \vec{k}_{0}
$$

Any beam may be decomposed in terms of such plane waves and it follows that no component of the linear momentum can exceed $\hbar k_{0}$ per photon. In particular, if we work in cylindrical polar coordinates then the azimuthal component of the momentum at any point in our light beam should be limited by

$$
p_{\phi} \leq p=\hbar k_{0}
$$

The angular momentum at a given radius $r$ in the $z$-direction is $j_{z}=r p_{\phi}$. If 
we associate this quantity with the $l \hbar$ carried by each photon then we are led to conclude that the azimuthal linear momentum per photon at distance $r$ from the beam axis will be $[1,2,4,5]$

$$
p_{\phi}=\frac{\hbar l}{r} .
$$

This expression predicts that the azimuthal component of the linear momentum increases without limit as the vortex lying on the the beam axis is approached. We are led to conclude that the local value for the azimuthal linear momentum (3) is in conflict with the inequality (2). This suggests that the field amplitude should be zero for

$$
r \leq \bar{r}=\frac{\hbar l}{\hbar k_{0}}=\frac{l \lambda}{2 \pi}
$$

where $\lambda$ is the wavelength. This conflicts with the known forms of the LaguerreGaussian and Bessel beams which have been shown to exhibit optical vortices, carry orbital angular momentum and all have a non-zero amplitude for $r \neq 0$ $[1,2,6,7]$.

The paradox has been presented using a geometrical argument based on a ray optics description, but the same conflict appears within a wave description. Consider a simple wave carrying orbital angular momentum:

$$
u(r, \phi, z)=u_{0}(r) e^{i l \phi} e^{i k_{z} z}, \quad l \neq 0 .
$$

The spatial frequencies of the central circular region, of radius $R$, are

$\tilde{u}(k, \theta, z)=\frac{1}{2 \pi} \int_{0}^{2 \pi} d \phi \int_{0}^{R} d r r u(r, \phi, z) e^{i \vec{k} \cdot \vec{x}}=i^{l} e^{i l \theta} \int_{0}^{R} d r r u_{0}(r) J_{l}(k r) e^{i k_{z} z}$,

where $k_{z}^{2}+k^{2}=k_{0}^{2}, \vec{x}=(r, \phi)(\vec{k}=(k, \theta))$ is the near (far) field position vector in polar coordinates, and $J_{l}$ is a Bessel function of the first kind [8]. Intense spatial components (6) require $k R \gtrsim l$, otherwise the very small value of the 
Bessel function leads to a near-vanishing integral. If $k>k_{0}$ then $k_{z}$ is forced to be imaginary so that the wave is evanescent and does not propagate. In order to have propagating components, therefore, we require $k_{0} R \gtrsim l$, which is again the inequality (4).

We should note that our inequality (4) has appeared before in two related problems. It was derived as a estimate of the minimum size of object to which orbital angular momentum could be transfered from a Laguerre-Gaussian beam

[9]. It is also related to an inequality derived for the maximum topological charge that can be contained within any given region [10]. The latter result was based on the observation that violation of the inequality would necessarily lead to the generation of evanescent waves and that the field would not then propagate.

\section{Propagation of a Bessel beam through a cir- cular aperture}

We can attempt a resolution of the paradox by isolating the part of the beam in the vicinity of the vortex and thereby examining its properties. In order to do so, we consider the propagation of a Bessel beam through a circular aperture centred on the beam axis. In analysing this problem it is sufficient to work within scalar wave theory as we are not concerned with the spin angular momentum associated with polarisation. It is not sufficient, however, to employ the paraxial approximation [11] as we need to allow for the possibility of evanescent waves, that it waves decaying exponentially in the propagation direction. For the same reason we should be cautions in using Fraunhofer diffraction as that is based on the propagation of Huygens's secondary waves [12]. We will see in the next Section, however, that simple diffraction theory can reproduce the essential 
features of the problem.

In scalar wave theory, the field $u$ with angular frequency $c k_{0}$ satisfies the Helmholtz equation

$$
\nabla^{2} u+k_{0}^{2} u=0 .
$$

Solution of this equation by means of separation of variables in cylindrical polar coordinates gives the Bessel beams

$$
u(r, \phi, z)=A e^{i k_{z} z} e^{i l \phi} J_{l}\left(k_{t} r\right) .
$$

Here $A$ is the amplitude and $k_{0}^{2}=k_{z}^{2}+k_{t}^{2}$. The azimuthal phase dependence $e^{i l \phi}$ means that such fields carry angular momentum $l \hbar$ per photon and, for $l \neq 0$ have an optical vortex centred at $r=0$. Bessel beams are also of interest as they propagate without changing their form [13].

We consider a Bessel beam of the form (8) propagating in the positive $z$ direction and incident on a circular aperture of radius $R$ placed in the $z=0$ plane and centred on $r=0$. Our task is then to calculate the form of the field propagating through the aperture, that is to find $u(r, \phi, z)$ for $z>0$. The circular aperture does not break the cylindrical symmetry of the problem and hence the angular momentum of the beam will be conserved and $u$ will retain its $e^{i l \phi}$ azimuthal dependence. A continuous and differentiable solution is obtained by considering transmitted and reflected waves generated at the plane of the aperture. It is convenient to express the transmitted wave for $z>0$ and the reflected wave for $z<0$ in terms of Bessel function solutions to the Helmholtz equation in the form

$$
\begin{gathered}
u_{\mathcal{T}}(r, \phi, z>0)=\int_{0}^{\infty} d k \mathcal{T}(k) e^{i \sqrt{k_{0}^{2}-k^{2}} z} e^{i l \phi} J_{l}(k r), \\
u_{\mathcal{R}}(r, \phi, z<0)=\int_{0}^{\infty} d k \mathcal{R}(k) e^{-i \sqrt{k_{0}^{2}-k^{2}} z} e^{i l \phi} J_{l}(k r),
\end{gathered}
$$


where $\mathcal{T}(k)$ and $\mathcal{R}(k)$ are transmission and reflection functions determined from the form of the field incident on the aperture. We note that the integral is evaluated over an infinite range of $k$-values. For $k<k_{0}$ a propagating field will result, but for $k>k_{0}$ the $z$-dependence will take the form of an exponential decay, $e^{-\sqrt{k^{2}-k_{0}^{2}}|z|}$ corresponding to evanescent waves. We find the transmission and reflection functions by requiring that $u+u_{\mathcal{R}}$, in Eqs. (8) and (10), and $u_{\mathcal{T}}$, Eq. (9), together with their first derivatives, match at $z=0$ :

$$
\begin{aligned}
& \mathcal{T}(k)=\frac{1}{2}\left(\frac{\sqrt{k_{0}^{2}-k_{t}^{2}}}{\sqrt{k_{0}^{2}-k^{2}}}+1\right) A k \int_{0}^{R} d r r J_{l}\left(k_{t} r\right) J_{l}(k r) \\
& \mathcal{R}(k)=\frac{1}{2}\left(\frac{\sqrt{k_{0}^{2}-k_{t}^{2}}}{\sqrt{k_{0}^{2}-k^{2}}}-1\right) A k \int_{0}^{R} d r r J_{l}\left(k_{t} r\right) J_{l}(k r) .
\end{aligned}
$$

The Fourier-Bessel integral in Eqs. (11-12) is [14]

$$
\int_{0}^{R} d r r J_{l}\left(k_{t} r\right) J_{l}(k r)=R \frac{k J_{l}\left(k_{t} R\right) J_{l-1}(k R)-k_{t} J_{l-1}\left(k_{t} R\right) J_{l}(k R)}{k_{t}^{2}-k^{2}} .
$$

In the limit of very large aperture radius $R$ this integral tends to $\delta\left(k-k_{t}\right) / k$, the reflection coefficient (12) vanishes and the whole wave is transmitted.

The intensity of the beam propagating through the aperture is obtained from the propagating part of the field (9). Within a scalar representation of the electromagnetic field [15], the flux of energy in the propagation direction is obtained by integrating over the transverse plane the $z$ component of the Poynting vector $\frac{i c^{2}}{2}\left[u \frac{\partial}{\partial z} u^{*}-c . c.\right]$. Hence the total power reaching the far-field is

$$
P^{F F}=\lim _{z \rightarrow \infty} \int d \phi \int d r r \frac{i c^{2}}{2}\left[u_{\mathcal{T}}(r, \phi, z) \frac{\partial}{\partial z} u_{\mathcal{T}}^{*}(r, \phi, z)-c . c .\right],
$$

where the limit serves to remove the evanescent contributions. This power depends on the aperture size $(R)$ and on the characteristics of the input beam $\left(l, k_{t}\right)$. It also depends on the total power incident on the aperture $P_{0}=$ $c^{2} \int d \phi \int_{0}^{R} d r r|u(r, \phi, 0)|^{2} \sqrt{k_{0}^{2}-k_{t}^{2}}$. It is sensible to remove the dependence on 


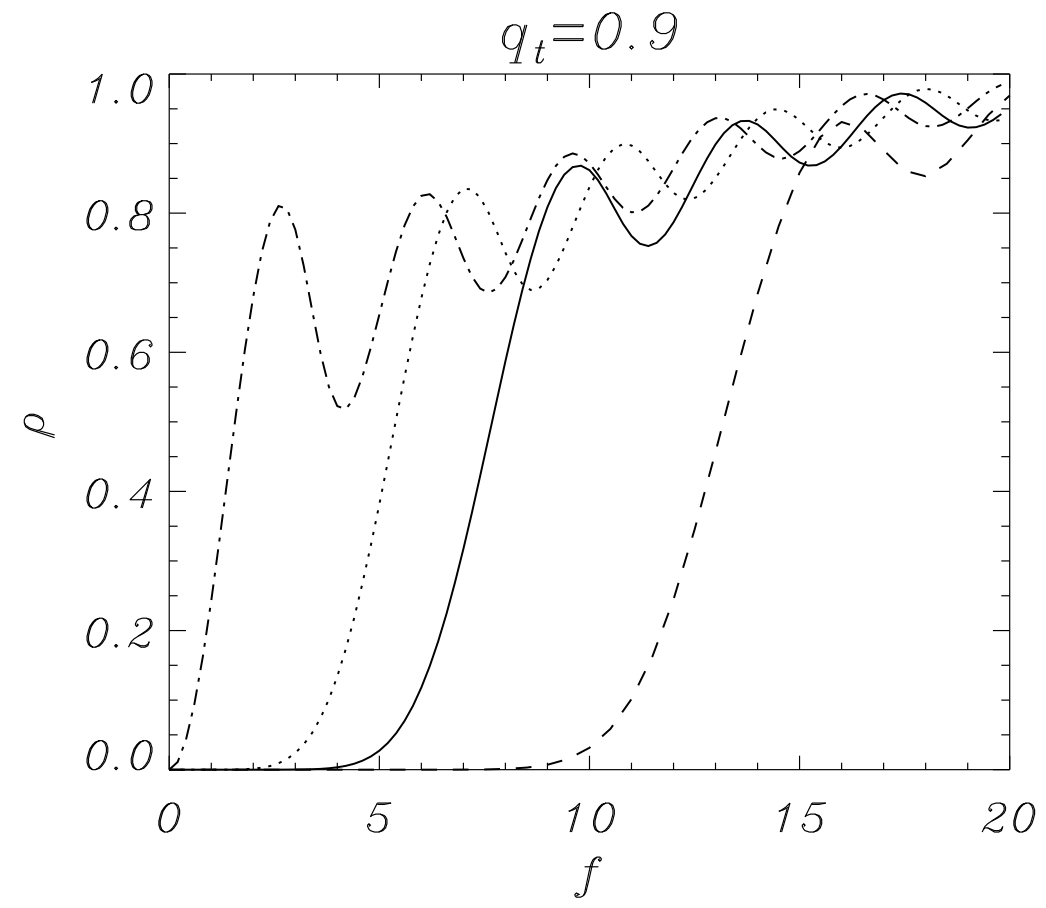

Figure 1: Ratio $\rho$ as a function of $f$ for $q_{t}=0.9$, and $l=0$ (dashed-dotted line), $l=3$ (dotted), $l=5$ (continuous), $l=10$ (dashed line).

the incident power by considering the power ratio

$$
\begin{aligned}
& \rho=\frac{P^{F F}}{P_{0}}=\frac{1}{\int_{0}^{1} r d r J_{l}^{2}\left(q_{t} r f\right)} \frac{1}{4} \\
& \int_{0}^{1} d q q\left[\frac{q J_{l}\left(q_{t} f\right) J_{l-1}(q f)-q_{t} J_{l-1}\left(q_{t} f\right) J_{l}(q f)}{q_{t}^{2}-q^{2}}\right]^{2}\left[\frac{\left(1-q^{2}\right)^{\frac{1}{4}}}{\left(1-q_{t}^{2}\right)^{\frac{1}{4}}}+\frac{\left(1-q_{t}^{2}\right)^{\frac{1}{4}}}{\left(1-q^{2}\right)^{\frac{1}{4}}}\right]^{2}
\end{aligned}
$$

that is the fraction of the power incident on the aperture that propagates to the far field. Here we have introduced the normalised quantities $q_{t}=k_{t} / k_{0}$ and $f=R k_{0}$ corresponding to the transverse wavenumber and the aperture radius respectively.

In Fig. 1 we plot the power ratio $\rho$ as a function of the aperture size $f$. For apertures that are very small compared with the input wave-length there 
is no light propagating and the power ratio tends to zero. It is important to note that the plotted quantity is the transmitted light normalized with the light incident on the aperture. For small apertures, of course, both quantities are small, but Fig. 1 represents only the propagating component of the diffracted wave. For small $f$ the aperture excites only very high frequency components with large transverse wavevector, transforming the propagating input wave in an evanescent wave.

The aperture size needed to obtain a propagating wave strongly depends on the orbital angular momentum carried by the wave. In Fig. 1 we see that beams carrying non-zero orbital angular momentum, $l \neq 0$, need larger apertures to propagate through than beams with $l=0$. This means that in order to transmit a propagating wave from the region around a vortex core we need an aperture with a radius that increases with the index $l$. For $f<l$ evanescent components are mostly excited after the aperture, while for $f>l$ a propagating wave is transmitted. We note that $f<l$ corresponds to selecting only the part of the beam satisfying the inequality (4). We can conclude that the field in the region described by our paradox does not propagate and comprises purely evanescent components.

The ratio Eq. (15) also depends on $q_{t}$, the focussing of the beam, as shown in Fig. 2. For very large apertures $(f)$ the whole beam propagates through the aperture and evanescent components are negligible so $\rho \rightarrow 1$. For weakly focussed beams (small $q_{t}$, black line in Fig. 2) larger apertures are needed to obtain such a complete transmission. In general the ratio $\rho$ is not a monotonic function of the aperture size. For $f q_{t} \gtrsim l$ the ratio $\rho(f)$ oscillates with frequency $\pi / q_{t}$. A simple explanation may be found by considering the profile of the incident beam. For $f \sim l / q_{t}$ the aperture cuts the incident beam where it is 


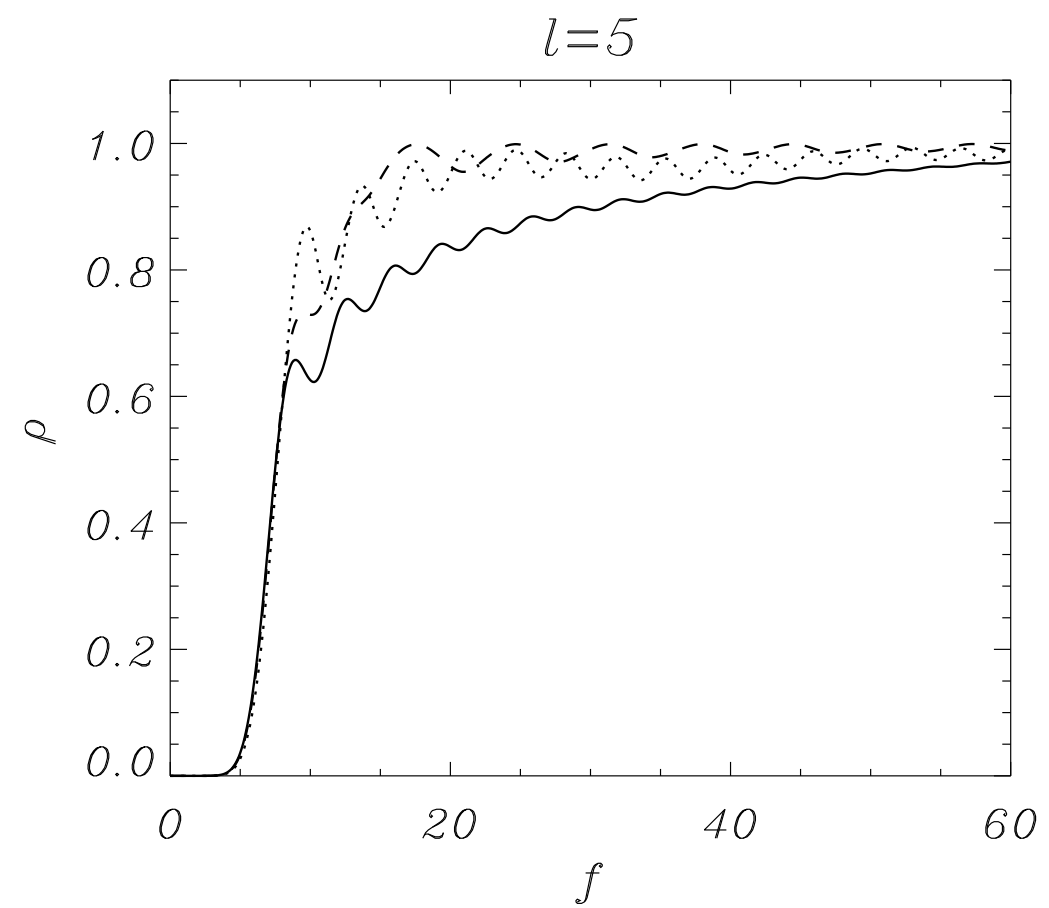

Figure 2: Ratio $\rho$ as a function of $f$ for $l=5$ and $q_{t}=0.9$ (dotted line), $q_{t}=0.5$ (dashed line), $q_{t}=0.1$ (continuous line). 
intense, along the inner bright ring of the Bessel beam, exciting high transverse spectral components that are evanescent. This leads to a local minimum of $\rho$ in Fig. 2. When the aperture size is increased to reach the next dark ring of the Bessel beam, evanescent components are only weakly excited giving a local maximum in Fig. 2. The periodicity $\pi / q_{t}$ in the ratio $\rho$ simply reflects the periodicity of the rings in the input intensity. Fig. 2 also shows that for small values of the aperture $f \lesssim l$ the power ratio $\rho$ obtained for different values of $q_{t}$ overlap. Indeed for the aperture sizes where the paradox occurs the transmission rate results largely independent of the focussing. We will further discuss this point in the next Section, in which we introduce a more qualitative description.

\section{$3 \quad$ Fraunhofer diffraction picture}

Eq. (15) has been obtained through the exact description of the diffraction of a wave through a circular aperture within a scalar theory. An approximate, but still qualitatively correct, picture can be given by neglecting the reflected wave, as in the more familiar Fraunhofer treatment of diffraction. The transmission function is now obtained only by requiring that $u$ and $u_{\mathcal{T}}$ equations (8) and (9) match continuously at $z=0$ :

$$
\mathcal{T}(k)=A k \int_{0}^{R} r d r J_{l}\left(k_{t} r\right) J_{l}(k r)
$$

Within this approximation, we take the total power to be $k_{0} c^{2} \int d \phi \int d r r|u|^{2}$, as in paraxial treatments. This simplified treatment can accommodate the excitation of evanescent wave components in the diffracted wave, by ignoring the requirement that $k_{0}^{2}=k^{2}+k_{t}^{2}$, as is usual in paraxial optics. The power ratio transmitted far from the aperture, not reached by evanescent components, is 
then

$$
\rho=\frac{1}{\int_{0}^{1} r d r J_{l}^{2}\left(q_{t} r f\right)} \int_{0}^{1} q d q\left[\frac{q J_{l}\left(q_{t} f\right) J_{l-1}(q f)-q_{t} J_{l-1}\left(q_{t} f\right) J_{l}(q f)}{q_{t}^{2}-q^{2}}\right]^{2} .
$$

This approximate expression is compared with the exact ratio Eq. (15) in Fig. 3, showing that the simplified model captures the relevant qualitative features of the exact description. In particular, it reproduce the turn-on in transmission at $f \sim l$ associated with the appearance of non-evanescent components. This is in accord with the simple qualitative argument based on spatial frequencies, presented in Sect. 1. The advantages of this simplified treatment are that it embodies the familiar paraxial approach usually used in describing optical angular momentum $[1,2]$, as well as providing the possibility of more immediate analytical approximations for the ratio $\rho$.

In Fig. 4 we plot the dependence of the ratio Eq. (17) on $q_{t}$, the focussing of the beam, clearly showing that for small values of the aperture $f \lesssim l$ the focussing is irrelevant, as anticipated in the previous Section. This means that it is the orbital angular momentum and not the degree of focussing of the beam that determines if any light will propagate through the aperture. This observation leads us to an approximate form for $\rho$, found by considering a weakly focussed beam $\left(q_{t} f \ll 2(l+1)\right)$. In this limit

$$
\rho \approx 2(l+1) \int_{0}^{1} d q J_{l+1}^{2}(f q) / q
$$

and we can evaluate the integral to give

$$
\rho \approx J_{l+1}^{2}(f)+2 \sum_{k=l+2}^{\infty} J_{k}^{2}(f) .
$$

For values of $f$ within the region associated with the paradox, we can further approximate this result by just its first term. This simple expression is a very good approximation, as can be seen in Fig. 5, being good also for large values 


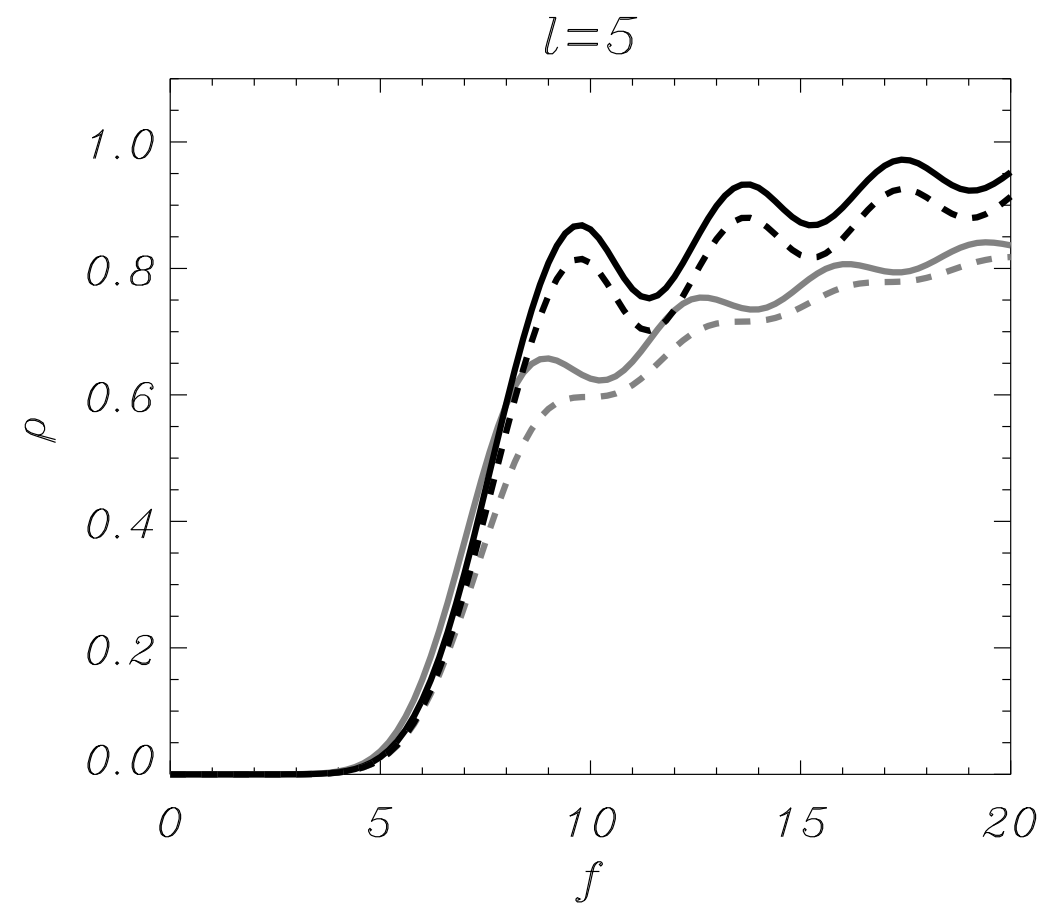

Figure 3: Comparison of the exact ratio Eq. (15) (continuous lines) and of the approximated expression Eq. (17) (dashed lines) for $l=5$. Grey curves correspond to weak focussing $\left(q_{t}=0.1\right)$, and black curves to strong focussing $\left(q_{t}=0.9\right)$. 


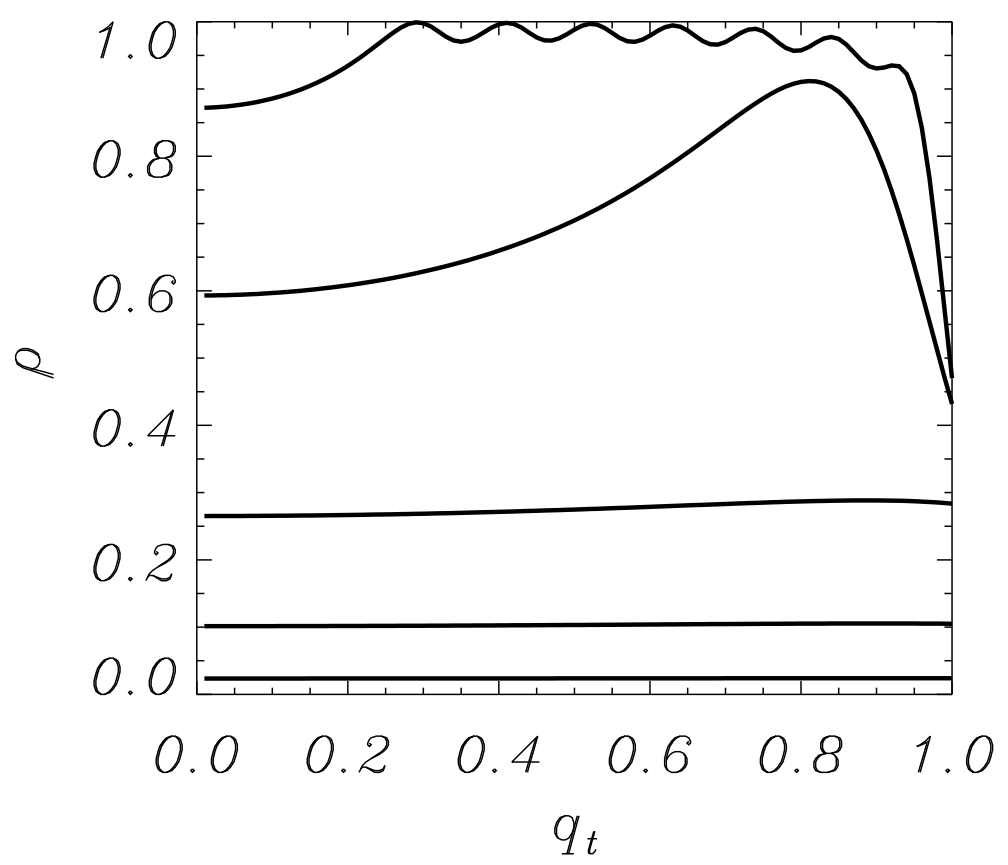

Figure 4: Ratio $\rho$ (17) for $l=5$ as a function of $q_{t}$. From the lowest line $f=5,6,7,10,30$. 


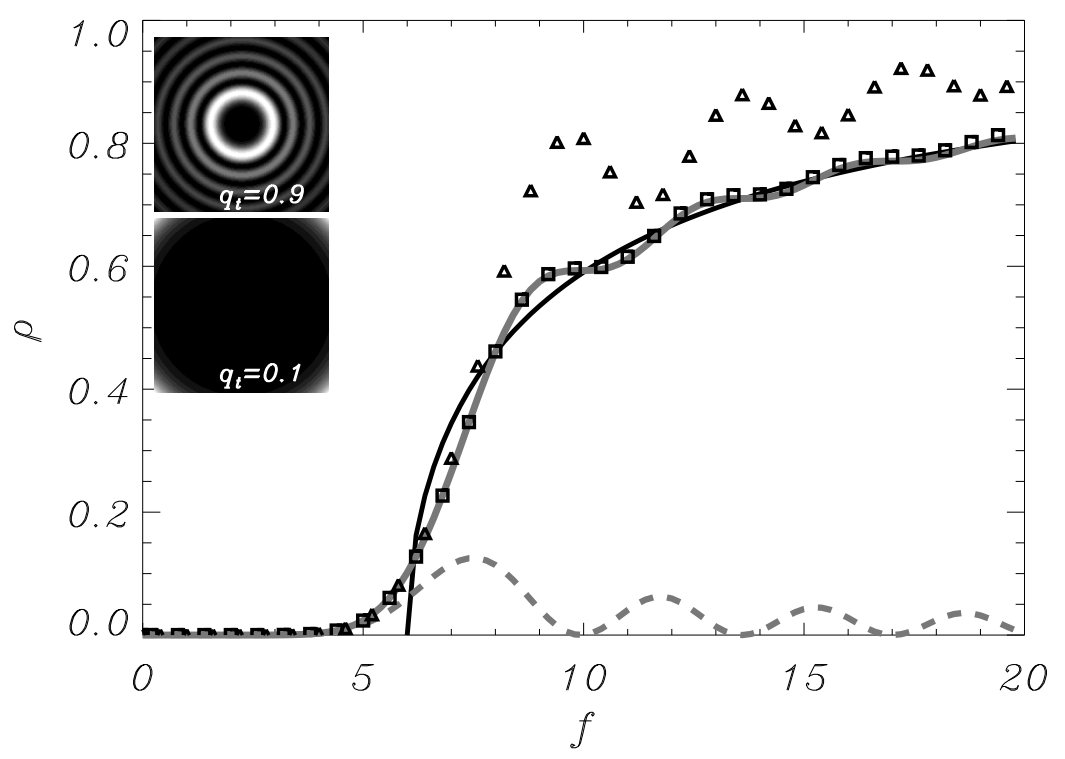

Figure 5: The ratio $\rho$ (17) as a function of $f$, for $l=5$ and $q_{t}=0.1$ (squares line), 0.9 (triangles line). The continuous grey line represents the approximation (19) and the dashed grey line is the first term in (19), $J_{6}^{2}(f)$. The approximation (21) is represented by the black line. The inserts show the intensity transverse profile of the input beam $J_{5}^{2}\left(k_{t} r\right)$ for $f=k_{0} R=5$, plotted in squares of size $-4<x, y<4\left(\frac{r}{R}=\sqrt{x^{2}+y^{2}}\right)$.

of $q_{t}$ and $f$. We conclude that the degree of focussing of the input Bessel beam is not a relevant factor to control the transmission of light through the aperture in the region of interest $(f \lesssim l)$. Here the relevant parameter is only the orbital angular momentum, associated with the index $l$, which fixes the power law near the vortex core.

A further approximation of Eq. (18) can be introduced in the case of large apertures. If we consider rather large values of $l$ the integrand in Eq. (18) can be approximated by high order Bessel beam expansions [17]. For $f>(l+1) / q$

$$
J_{l+1}^{2}(f q) \approx 2 \cos ^{2}(\alpha)\left[\pi \sqrt{(f q)^{2}-(l+1)^{2}}\right]^{-1}
$$


where $\alpha$ is a complicated function of $f q$ and $l+1$. For $f<(l+1) / q$ the integrand decays rapidly and we neglect the integral in this region. If we approximate $\cos ^{2}(\alpha)$ by its average value $(1 / 2)$ then the integral (18) can be evaluated to give

$$
\rho \approx\left[1-\frac{2}{\pi} \arcsin \left(\frac{l+1}{f}\right)\right] \Theta(f-l-1)
$$

This expression is compared with the ratio $\rho$ (17) in Fig. 5. We note that Eq. (21) allows for the identification of the relevant scaling parameter $\frac{l+1}{f}$, confirming that larger apertures are needed to transmit beams carrying orbital angular momentum, according to a linear relation between $l$ and $f$.

We have found that when isolated by means of an aperture, the field in the vicinity of a vortex core does not propagate. We might reasonably ask where this light comes from. The answer, of course, is that in propagation of the whole beam the field near to the vortex core is the result of diffractive contributions from the whole field in any previous plane. The Bessel beam does not diffract but this does not mean that we can associate the field in any given region of a plane with the corresponding region of earlier planes. By virtue of Babinet's principle [12] the beam (8) can be written as the superposition of two waves diffracted by complementary apertures, our circular aperture of radius $R$ and a circular mask of the same radius. The component diffracted by an hole of radius $R<\bar{r}$, Eq. (9), is evanescent. The component associated with the circular mask, therefore comprises our Bessel beam minus an evanescent component that cancels with that associated with the circular aperture. It follows that a beam with $l \neq 0$ impinging on an opaque circular object with radius $R$ would be completely reconstructed after a free propagation distance such that the evanescent components vanish [16]. 


\section{Resolution and conclusions}

We have presented a paradox for a beam carrying orbital angular momentum, arising from the comparison of the local dependence of the azimuthal linear momentum (3) with the momentum for a plane wave (2). This led us to question the possibility of having light within a finite radius of the vortex core (4). We have shown that no propagating light can be isolated in this region, as any attempt to select it inevitably gives rise to evanescent waves. Nevertheless the intensity in this region is not zero. We are now in a position to resolve our paradox.

We start by noting that the paradox involves localised angular momentum and momentum. Within a wave theory, however, these should be discussed in terms of densities. The densities of momentum and angular momentum have been calculated for Bessel beams[6, 7] and for Gauss-Laguerre beams [1, 4]. In both cases the azimuthal linear momentum density was shown to be inversely proportional to the distance from the beam axis. This is not a problem for the momentum density as it is not constrained to equal $\hbar k_{0}$ and does, in fact, depend on the position within the beam. Thus there is no problem with a local violation Eq. (2), as long as we do not attempt to violate it for the whole beam.

If we try to isolate a region of the beam in which the inequality (2) is violated then we inevitably excite evanescent waves. Such waves have an imaginary value of the $z$-component of the wavevector and so can tolerate values of $k_{\phi}>k_{0}$. Our calculation of the propagation of Bessel beams through a small circular aperture is a direct demonstration of this point.

We conclude by noting that our analysis is based only on the properties of waves satisfying the Helmholtz equation. No other features specific to light have been employed. Hence we would expect that the ideas presented here should 
apply to all wave forms including acoustic waves and electron wavefunctions.

\section{Acknowledgements}

We are grateful to Sir Michael Berry for his encouragement and also for the elegant approximation that led to Eq. (21). We thank Johannes Courtial, Jonathan Leach, Graham Gibson, Les Allen and Mikhail Vasnetsov for helpful and entertaining discussions. This work was supported by the Engineering and Physical Sciences Research Council (GR/S03898/01).

\section{References}

[1] Allen, L., BeiJersbergen, M. W., SPREEUW, R. J. C., and WOERDMAN, J. P., 1992, Phys. Rev. A, 40, 8185.

[2] Allen, L., BARnETT, S. M., and PADGETT, M. J., 2003, Optical Angular Momentum (Bristol: Institute of Physics).

[3] NYE, J. F., 1999, Natural Focussing and Fine Structure of Light (Bristol: Institute of Physics) and references therein.

[4] PADGetT M. J., and ALlEN, L., 1995, Opt. Commun., 121, 36.

[5] ALlEn, L, and PADGETT, M. J., 2000, Opt. Commun., 184, 67.

[6] BARnetT, S. M, and ALLEN, L., 1994, Opt. Commun., 110, 670.

[7] VOLKE-SEPULVEDA, K, GARCHÉS-CHAVÉZ V., CHAVÉZ-CERDA, J, ARLT, J., and DHOLAKIA, K., 2002, J. Opt. B: Quantum Semiclass Opt., 4, S82.

[8] WATSON, G. N., 1995, A Treatise on the Theory of Bessel Functions 2nd ed. (Cambridge: Cambridge University Press). 
[9] COURTiAl J., and PADGETT, M. J., 2000. Opt. Commun., 173, 269.

[10] ROUX, F. S., 2003, Opt. Commun., 223, 31.

[11] SIEGMAN, A. E., 1986, Lasers (Mill Valley: University Science Books).

[12] BORN, M., and WOLF, E., 1999, Principles of Optics 7th ed. (Cambridge: Cambridge University Press).

[13] DURnin, J., MiCELI, J. J., and EBERLY, J. H., 1987, Phys. Rev. Lett., $\mathbf{5 8}, 1499$.

[14] GRAY, A., MATTHEWS, G. B., and MACROBERT, T. M., 1922, A Treatise on Bessel Functions (London: Macmillan).

[15] GREEN, H. S. and WOLF, E., 1953, Proc. Phys. Soc. A 66, 1129; WOLF E., 1959, Proc. Phys. Soc. 74, 269; ROMAN P., 1959, Proc. Phys. Soc. 74, 281.

[16] BOUCHAL, Z., WAGNER, J., and CHLUP, M., 1998, Opt. Commun. 151, 207.

[17] See Eq. (9.3.3) and Eq. (9.3.7) in ABRAMOWITZ, M. and STEGUN I. A., 1970, Handbook of mathematical functions (Dover, New York). 